\title{
Murciélagos del área urbana en la ciudad de Montería, Córdoba - Colombia
}

\author{
Urban Bats from the City of Monteria, Cordoba - Colombia
}

Jesús Ballesteros C, ${ }^{1 *}$ Ph.D, Javier Racero-Casarrubia, ${ }^{2}$ M.Sc.

${ }^{1}$ Universidad de Córdoba, Facultad de Ciencias Básicas, Departamento de Biología. Grupo Investigación Biodiversidad Unicórdoba. Montería, Colombia. ${ }^{2}$ Parque Natural Nacional Paramillo. Grupo de Investigación Biodiversidad Unicórdoba. *Correspondencia: jballescor@yaahoo.com

Recibido: Julio de 2011; Aceptado: Enero de 2012.

\section{RESUMEN}

Objetivo. Conocer la riqueza de especies de murciélagos del área urbana de la ciudad de Montería. Materiales y métodos. Durante el período de enero a junio de 2007, se realizaron capturas de murciélagos utilizando cinco redes de nieblas $(12 \times 2 \mathrm{~m})$. Se realizaron trece muestreos en varios sitios de la ciudad, abriendo las redes desde las 18:00 a las 24:00 horas, con un esfuerzo de 524 horas-red/ noche. Resultados. De 604 individuos capturados se identificaron 24 especies de murciélagos. La mayor abundancia relativa se presentó en las especies Artibeus planirostris (54\%), Artibeus lituratus (11.2\%) Sturnira lilium (7.4\%) y Glossophaga soricina (4.2\%). Conclusiones. El ensamblaje de murciélagos en el área urbana estuvo representado por los gremios insectívoros, frugívoros, nectarívoros, omnívoros y piscívoros. La familia Phyllostomidae presentó la mayor diversidad de especies.

Palabras clave: Clima tropical, ecología, mamíferos, quirópteros (Fuente: MeSH).

\begin{abstract}
Objective. To identify the abundance of bat species in urban areas of the city of Monteria. Materials and methods. During the period from January to June 2007, collections of bats were made using five $(12 \times 2 \mathrm{~m})$ mist nets. Thirteen surveys were conducted at several sites in the city, opening up the networks from 18:00 to 24:00 hours, with an effort of 524 hours-net/night. Results. A total of 604 catches, with 24 species of bats identified. The highest relative abundance of species occurred in Artibeus planirostris (54\%), Artibeus lituratus (11.2\%), Sturnira lilium (7.4\%) and Glossophaga soricina $(4.2 \%)$. Conclusions. The assembly of bats in urban area was represented by insectivores, frugivores, nectarivores, omnivores and piscivores. The family Phyllostomidae had the highest species diversity.
\end{abstract}

Key words: Chiroptera, ecology, mammals, tropical climate (Fuente: MeSH). 


\section{REVISTA MVZ CÓRDOBA • Volumen 17(3) Septiembre - Diciembre 2012}

\section{INTRODUCCIÓN}

Los murciélagos, únicos mamíferos capaces de volar, tienen amplia distribución en el planeta son especialmente diversos en el Neotrópico y se desempeñan en una gran variedad de nichos tróficos (1). Con más de 1100 especies a nivel mundial (2) y 220 especies en el Neotrópico (3), los murciélagos constituyen el segundo orden taxonómico más diverso entre los mamíferos. Colombia con 197 especies de murciélagos $(4,5)$, ocupa el primer lugar en América y segundo en el mundo en cuanto a la diversidad de quirópteros (6). Para el departamento de Córdoba se han identificado ocho familias, 37 géneros y unas 66 especies de murciélagos.

Estos organismos cumplen una importante función en procesos ecológicos de los bosques tropicales, especialmente como polinizadores de plantas $(7)$, dispersores de semillas $(8,9)$ y controladores de poblaciones de insectos plaga $(10,11)$, un aspecto beneficioso para la agricultura al consumir millones de insectos plaga en los cultivos cada noche (12). El desconocimiento de la ecología, ha resultado perjudicial para la conservación de la diversidad de murciélagos; siendo poco valorados por la comunidad en general, que por sus hábitos nocturnos, se estigmatizan como animales dañinos y vectores de enfermedades.

En Colombia, aunque se cuenta con numerosos estudios de murciélagos en áreas rurales, especialmente sobre diversidad de especies, ecología, sistemática, distribución e implicaciones para la salud humana; es poca la literatura disponible que documente aspectos ecológicos de los murciélagos en ecosistemas urbanos. La generación de información científica sobre murciélagos en áreas urbanas es de gran importancia para futuros planes de conservación y manejo. Un estudio integral de los murciélagos en ambientes urbanos puede ayudar a eliminar algunos prejuicios; especialmente de aquellas especies que generan molestias al cohabitar con el hombre y que algunas veces, puede presentar problemas de salud pública, como es la histoplasmosis, una enfermedad causada por un hongo microscópico del suelo (Histoplasma capsulatum), que puede estar presente en los excrementos de murciélagos, además de su papel como transmisores de enfermedades zoonóticas como la rabia, la leptospirosis, la histoplasmosis y la encefalitis equina (13).

Estudios de murciélagos asociados a áreas urbanas en Colombia son escasos y para Córdoba, sólo un trabajo se refiere al tema (14), quizás debido a que la mayoría de estudios biológicos se orientan casi exclusivamente al sector rural. Es importante reconocer que los ecosistemas urbanos, al estar inmersos en una matriz del paisaje contienen importante diversidad de especies faunísticas, muchas veces desconocidas, en especial aquellas especies poco carismáticas como los quirópteros. Considerando la escasa literatura disponible que documente aspectos ecológicos de los murciélagos de las áreas urbanas en Colombia, este artículo, tiene como objetivo aportar información para llenar los vacíos de conocimientos sobre la composición del ensamble de murciélagos del área urbana de la ciudad de Montería.

\section{MATERIALES Y MÉTODOS}

Área de estudio. El trabajo se desarrolló en el área urbana del municipio de Montería, departamento de Córdoba, al noroccidente de Colombia (Figura 1). Esta región presenta un clima cálido, con temperatura media de $28^{\circ} \mathrm{C}$ y una precipitación media anual de $1200 \mathrm{~mm}$, con dos épocas bien marcadas; una época lluviosa (mayo-noviembre) y una época seca (diciembre-abril). El territorio del área urbana de Montería está dividido en nueve comunas, que se extiende en la parte media del Valle del Río Sinú en un paisaje plano de origen fluvio-lacustre y colinas. El área municipal comprende cerca de 312.200 ha y un perímetro urbano de 4.300 ha. El patrón de uso del suelo es de ganadería extensiva con pastos manejados, con predominio de parches de pastizales naturales y un sector bien definido de parches de cultivos en las áreas más próximas al río Sinú. En los alrededores

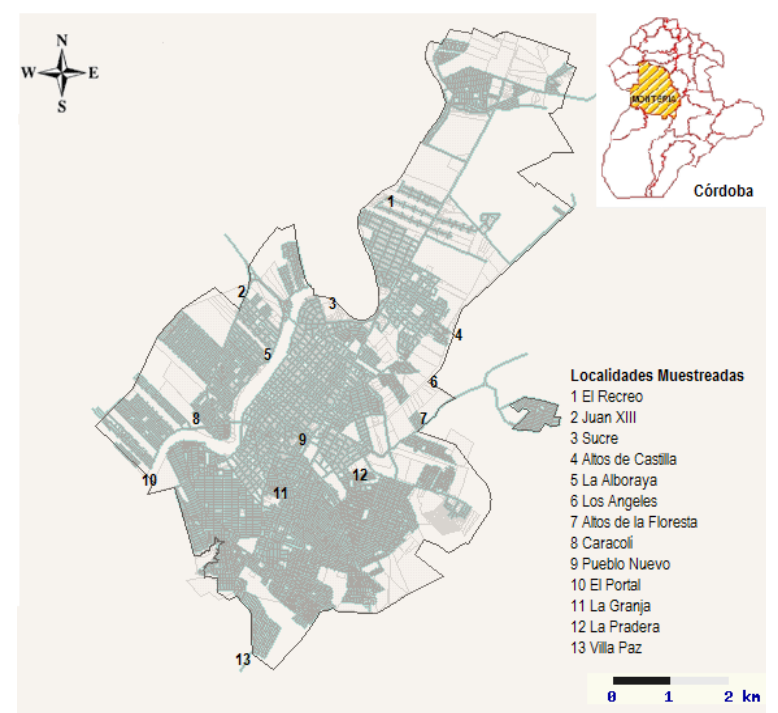

Figura 1. Localización del área de estudio y distribución de los sitios de muestreo de murciélagos en la ciudad de Montería, Córdoba. 
de la ciudad de Montería la vegetación original ha sido completamente degradada a pequeños fragmentos de bosque secundario o bosque de galería.

Métodos. Entre los meses de enero a julio del 2007, se realizaron muestreos de murciélagos en 13 sitios del área urbana de Montería. Se utilizaron cinco redes de niebla $(12 \times 2.5 \mathrm{~m})$, ubicadas al azar, abiertas desde las 18:00 hasta las 24:00 horas, y monitoreadas cada 30 minutos. Los murciélagos capturados se depositaron en bolsas de tela y luego procesados. Para la identificación de las especies, a todos los especímenes capturados se les tomó las medidas morfométricas estándar (longitud total, longitud de: cola, oreja, trago, hoja nasal, antebrazo, envergadura, pata con uña, pata sin uña, y calcáneo) con calibrador digital precisión $0.01 \mathrm{~mm}$. El peso del cuerpo se tomó con balanza electrónica de precisión $0.1 \mathrm{~g}$, previamente calibrada.

Para la identificación de las especies se utilizaron claves taxonómicas $(15,16)$ y las descripciones de Gardner (7); la taxonomía de géneros y especies sigue a Wilson y Reeder (17). La identificación de gremios alimenticios se realizó con base en las categorías: insectívoros, frugívoros, nectarívoros, carnívoros, piscívoros y hematófagos (18). Se calculó el éxito de captura como el número de individuos capturados respecto al esfuerzo de muestreo (individuos-noche/horas-red). Se elaboraron curvas de acumulación de especies usando el programa estadístico EstimateS Versión 7.5.1.

Se realizó una colección de referencia mediante especímenes voucher macho y hembra de cada especie; cuyo material biológico está depositado en la colección del Instituto de Ciencias Naturales (ICN) de la Universidad Nacional de Colombia (especímenes aún no catalogados). La colección fue realizada bajo el permiso de colecta biológica expedido por la Corporación Autónoma Regional de los Valles del Sinú y San Jorge (CVS) resolución No 1147 de agosto 2 de 2007.

\section{RESULTADOS}

Se capturaron 604 individuos con un éxito de captura de 1.15 individuos/horas-red. Se identificaron 24 especies de murciélagos comprendidos en 14 géneros y 5 familias. La familia con mayor riqueza de especies fue Phyllostomidae con 554 individuos (12 especies), seguida de Molossidae con 24 individuos (4 especies). Las familias Noctilionidae y Emballonuridae estuvieron representadas por un sólo individuo (Tabla 1). El estimador de riqueza CHAO 1 indica una representatividad del muestreo del $98 \%$ (Figura 2).

Tabla 1. Lista de especies de murciélagos y frecuencia de captura en el área urbana de Montería, Departamento de Córdoba - Colombia.

\begin{tabular}{|c|c|c|c|c|c|}
\hline \multirow{2}{*}{$\begin{array}{c}\text { Familia/ } \\
\text { Subfamilia }\end{array}$} & \multirow{2}{*}{ Especie } & \multirow{2}{*}{ Gremio* } & \multicolumn{2}{|c|}{ \# capturas/sexo } & \multirow{2}{*}{ Total capturas } \\
\hline & & & Machos & Hembras & \\
\hline \multicolumn{6}{|l|}{ Phyllostomidae } \\
\hline \multirow[t]{5}{*}{ Stenodermatinae } & Artibeus planirostris (Spix, 1823) & $\mathrm{F}$ & 132 & 191 & 323 \\
\hline & Artibeus lituratus (Olfers, 1818) & $\mathrm{F}$ & 25 & 42 & 67 \\
\hline & Uroderma bilobatum Peters, 1866 & $\mathrm{~F}$ & 7 & 3 & 10 \\
\hline & Sturnira lilium (E. Geoffroy, 1810) & $\mathrm{F}$ & 15 & 41 & 56 \\
\hline & Sturnira sp. & $\mathrm{F}$ & 3 & 10 & 13 \\
\hline \multirow[t]{2}{*}{ Phyllostominae } & Phyllostomus discolor Wagner, 1843 & $\mathrm{O}$ & 6 & 8 & 14 \\
\hline & Phyllostomus hastatus (Pallas, 1767) & $\mathrm{O}$ & 1 & 3 & 4 \\
\hline \multirow[t]{3}{*}{ Glossophaginae } & Anoura sp & $\mathrm{N}$ & 3 & 5 & 8 \\
\hline & Glossophaga soricina (Pallas, 1766) & $\mathrm{N}$ & 10 & 17 & 27 \\
\hline & Glossophaga longirostris Miller, 1898 & $\mathrm{~N}$ & 3 & 18 & 21 \\
\hline \multirow[t]{2}{*}{ Carollinae } & Carollia castanea H. Allen, 1890 & $\mathrm{~F}$ & 3 & 6 & 9 \\
\hline & Carollia perspicillata (Linnaeus, 1758) & $\mathrm{F}$ & 2 & 0 & 2 \\
\hline \multicolumn{6}{|l|}{ Molossidae } \\
\hline \multirow[t]{4}{*}{ Molossinae } & Molossops temmincki (Burmeister, 1854) & I & 3 & 2 & 5 \\
\hline & Molossus molossus (Pallas, 1766) & I & 2 & 6 & 8 \\
\hline & Molossus currentium Thomas, 1901 & I & 4 & 6 & 10 \\
\hline & Eumops bonariensis (Peters, 1874) & I & 0 & 1 & 1 \\
\hline \multicolumn{6}{|l|}{ Emballonuridae } \\
\hline Emballonurinae & Saccopteryx bilineata (Temminck, 1838) & I & 0 & 2 & 2 \\
\hline \multicolumn{6}{|l|}{ Vespertilionidae } \\
\hline \multirow[t]{3}{*}{ Myotinae } & Myotis nigricans (Schinz, 1821) & I & 1 & 9 & 10 \\
\hline & Myotis albescens (E. Geoffroy, 1806) & I & 10 & 0 & 10 \\
\hline & Eptesicus brasiliencis (Desmarest, 1819) & I & 1 & 0 & 1 \\
\hline Noctilionidae & Noctilio albiventris Desmarest, 1818 & $\mathrm{P}$ & 3 & 0 & 3 \\
\hline TOTAL & & & 234 & 370 & 604 \\
\hline
\end{tabular}




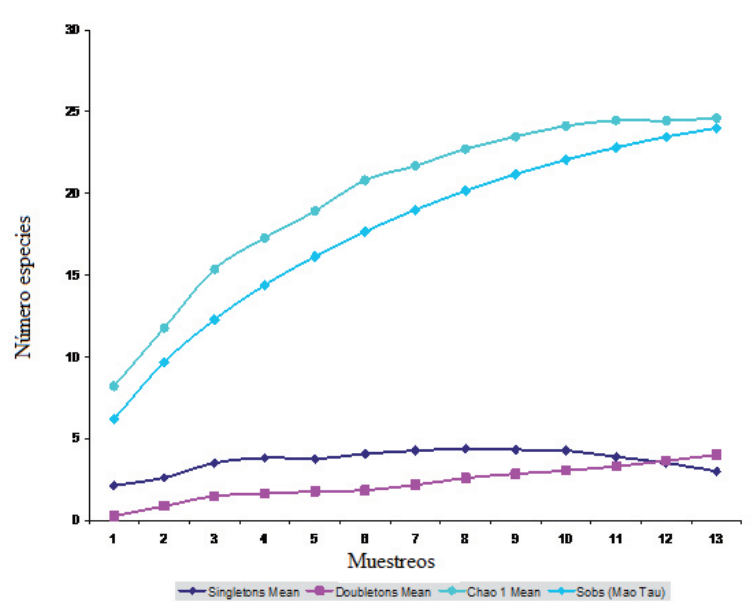

Figura 2. Curva de acumulación de especies de murciélagos capturados en el área urbana de la ciudad de Montería.

El ensamblaje de murciélagos para el área urbana de Montería, según gremios alimentarios, estuvo integrada por cinco categorías, dentro de las cuales los murciélagos insectívoros (38\%) presentaron mayor riqueza de especies, seguido por frugívoros, nectarívoros, omnívoros y pescadores (Figura 3). La familia Phyllostomidae presentó la mayor riqueza de especies, siendo Artibeus planirostris (Figura 4a) la más abundante; mientras que Molosus molossus (Figura 4b) fue la especie con mayor abundancia asociada a sitios de construcciones y techos de las viviendas urbanas.

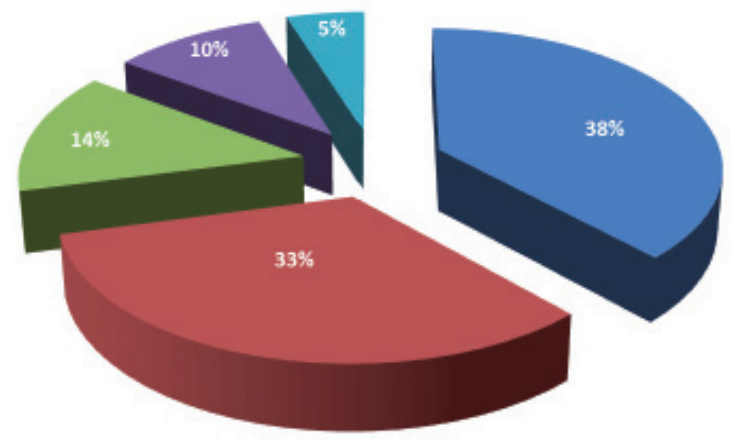

E Insectivoros = Frughworos $=$ Nectarivoros $=$ Omnivoros $=$ Piscivoros

Figura 3. Composición porcentual de la estructura trófica del ensamblaje de murciélagos en el área urbana de la ciudad de Montería, Córdoba.

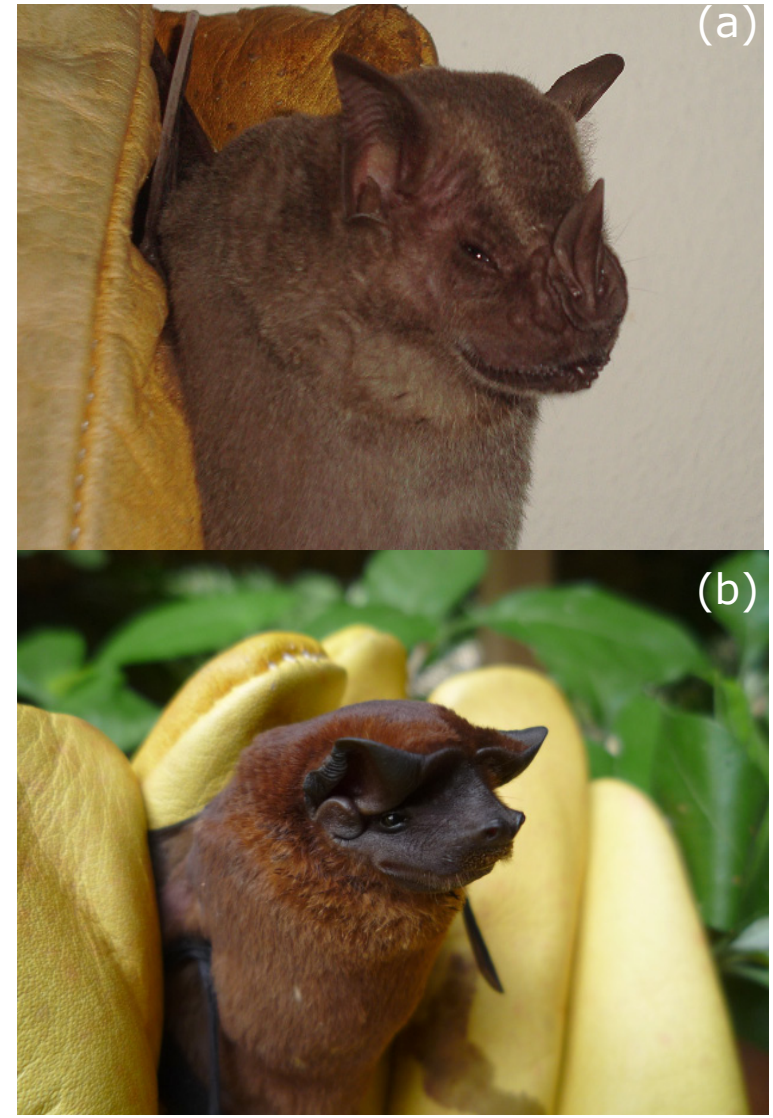

Figura 4. (a) Superior, murciélago frutero (Artibeus planirostris). (b) inferior, murciélago cola de ratón (Molossus molossus) capturados en el área urbana de la ciudad de Montería, Colombia.

\section{DISCUSIÓN}

Las especies reportadas para el área urbana de la ciudad de Montería equivalen al $51 \%$ de los murciélagos registrados para la zona costanera del departamento de Córdoba (19), y casi el doble número de especies reportadas por Muñoz-Saba (20) en muestreos realizados en las áreas rurales de Betancí y Martinica en el municipio de Montería; quizás con un mayor esfuerzo de muestreo, la riqueza se vea aumentada. En contraste con otras áreas urbanas de Colombia, se registró un número mayor de especies comparado con 6 especies reportadas por Alberico et al (21) para la ciudad de Santiago de Cali; 12 especies fueron registradas por Sampedro et al (22) para el área urbana de Sincelejo, donde Molossus molossus constituyó la especie más abundante ( $78 \%$ de las capturas). En otro estudio, el mismo autor registra el $42.3 \%$ de infestación por $M$. molossus en viviendas en regular y mal estado en el área urbana de Sincelejo (23). El papel ecológico que desempeña $M$. molossus como controlador de 
insectos parece tener importancia, al consumir insectos nocturnos, en su mayoría considerados insectos plaga. Desde el punto de vista de salud pública en Colombia y otras partes del mundo, M. molossus ha sido encontrado positivo para el virus de la rabia (24).

Este estudio muestra que el área rural y el área urbana de Montería comparten especies como: M. molossus, A. planirostris, G. longirostris, G. soricina, S. lilium, U. bilobatum. La mayor riqueza de especies en familia Phyllostomidae, es coherente con lo encontrado en otro estudio para el norte de Colombia (25); los murciélagos de este grupo son consideradas especies indicadores de hábitats intervenidos, en especial los géneros Carollia, Sturnira y Uroderma $(26,27)$. La abundancia dominante de $A$. planirostris concuerda con la característica generalista de la especie en cuanto a dieta y uso de hábitat (28).

Entre los murciélagos insectívoros que presentaron una dominancia del $38 \%$ de las capturas para el área urbana de Montería, se encuentra la familia Molossidae, segundo grupo más abundante, después de Phillostomidae, a pesar de no haber elevado redes a más de tres metros, condición que disminuye la posibilidad de capturar especies de vuelo alto. Estos resultados concuerdan con los obtenidos en estudios para áreas urbanas (29), donde aprovechan como sitios de forrajeo la presencia de luces artificiales, que atraen los insectos (30).

En este trabajo se identificaron algunos refugios en cielo raso de viviendas y debajo de puentes, lo que permitió capturar cuatro especies de la familia Molossidae, donde M. molossus fue más abundante. La presencia de M. molossus generalmente se le asocia a sitios con intervención antrópica, se le encuentra formando colonias en construcciones y debajo de los techos; esta especie se ha reportado como muy abundante en zonas urbanizadas al norte y suroccidente de Colombia $(21,23)$.

Los murciélagos frugívoros con el $33 \%$ de las capturas, es el segundo gremio más abundante en el área de estudio, donde es común observar que murciélagos del género Artibeus utilicen diferentes frutos de almendro (Terminalia catappa), especie muy apetecida por los murciélagos, y el níspero (Manilkara sapota), entre otras especies de Sapotaceae y Lauraceae, cuyos frutos también son consumidos por los murciélagos, dispersando sus semillas (31).

Es indiscutible la importancia de los murciélagos como dispersores de semillas y controladores de plagas $(9,12)$, por lo que es necesario promover el conocimiento de su diversidad y su papel funcional. Entender su ecología evitaría su exterminio, especialmente de aquellas especies de murciélagos que acostumbran a emplear como sitios de refugio el cielo raso de las casas, arboles en patios, hendiduras entre paredes o techos de palma. Algunos investigadores sugieren establecer hogares artificiales ubicados en parques, fuera de las viviendas para evitar este tipo de conflictos; sin embargo, su éxito es discutible porque los murciélagos irán donde ellos estimen conveniente, con el agravante que múltiples proyectos urbanísticos destruyen cada vez más los pocos espacios naturales en la periferia de la ciudad.

Se recomienda involucrar a la comunidad general en aspectos relacionados con la conservación de los murciélagos mediante el desarrollo de procesos de educación ambiental, desde la academia local hacia las comunidades. Tal proceso se puede realizar mediante talleres, programas radiales y afiches que den a conocer la importancia ecológica de los murciélagos en los ecosistemas.

En conclusión, el ensamblaje de murciélagos en el área urbana estuvo representado por los gremios insectívoros, frugívoros, nectarívoros, omnívoros y piscívoros. La familia Phyllostomidae presentó la mayor diversidad de especies.

\section{Agradecimientos}

Al Dr. Jairo Pérez-Torres, profesor de la Universidad Javeriana por su colaboración en la revisión y comentarios al documento. A Katia Reyes y Cristian González por su participación en trabajo de campo y laboratorio.

\section{REFERENCIAS}

1. Meyer C, Frund J, Pineda-Lozano W, Kalko E. Ecological correlates of vulneravility to fragmentation in Neotropical bats. J Appl Ecol 2008; 45:381-391.
2. Simmons NB. Order Chiroptera. En: Wilson DE \& Reeder DM (Eds.), Mammal Species of the World. Baltimore: Johns Hopkins University Press 2005; 312-529. 
3. Voss RS. Mammals of South America, Volume 1 Marsupials, Xenarthrans, Shrews, and Bats. Book Review. J Mammal 2009; 90(2):521-523.

4. Alberico $M$, Cadena A, Hernández-C J, Muñoz-S Y. Mamíferos (Synapsida: Theria) de Colombia/ mammals of Colombia. Biota Colombiana $2000 ; 1(1): 45-75$.

5. Mantilla-Meluk $H$, Jiménez-Ortega $A M$, Baker RJ. Phyllostomid Bats of Colombia: annotated Checklist, distribution and Biogeography. Texas: The Texas Tech University 2009.

6. Gardner AL. Mammals of South America, Volume 1. Marsupials, Xenarthrans, Shrews and Bats. Chicago: University of Chicago Press; 2007.

7. Arias-Coyotl E, Stoner K, Casas A. Effectiveness of Bats as Pollinators of Stenocereus stellatus (Cactaceae) in Wild, Managed in situ, and Cultivated Populations in La Mixteca Baja, Central Mexico. Am J Bot 2006; 93(11):1675-83.

8. Estrada-Villegas S, Pérez-Torres J, Stevenson P. Dispersión de semillas por murciélagos en un borde de bosque montano. Sociedad Venezolana de Ecología, Ecotropicos 2007; 20(1):1-14.

9. Lobova TA, Kulen K, Geiselman K, Mori SA. Seed dispersal by bat in the neotropics. New York: New York Botanical Garden; 2009.

10. Patterson BD, Willig MR, Stevens RD. Trophic strategies, niche partitioning, and patterns of ecological organization. En: Kunz TH \& Fenton MB (Eds.). Bat Ecology. Chicago: University of Chicago Press; 2003.

11. Cleveland $C J$, Betke $M$, Federico P, Frank JD, Hallam TG, Horn J, et al. Economic value of the pest control service provided by Brazilian free-tailed bats in south-central Texas. Front Ecol Environ 2006; 4:238-243.

12. Kalka MB, Smith AR, Kalko EKV. Bats limit arthropods and herbivory in a tropical forest. Science 2008; 320:71-71.

13. Núñez M, Aldaz JJ, Escobar H, Cuadros ME. Primer hallazgo de rabia silvestre en el murciélago insectívoro Eptesicus brasiliensis en Cali, Colombia. Rev Asoc Col Cienc Biol 2001; 12:57-61.
14. Suárez-Villota EY, Racero-Casarrubia J, Guevara G, Ballesteros J. Evaluación ecológica rápida de los quirópteros del parque ecológico de Montelíbano, Córdoba, Colombia. Trop Cons Sci 2009; 2(4):437-449.

15. Fernández $B$, Guerrero $R$, Lord $R$, Ochoa J, Ulloa G. Mamíferos de Venezuela, Lista y claves para su identificación. Museo del Instituto de Zoología Agrícola (Ed.). Caracas: Universidad Central de Venezuela; 1988.

16. Linares O. Mamíferos de Venezuela. Venezuela. Venezuela: Sociedad Conservacionista Audubon; 2000.

17. Wilson DE, Reeder DM (Eds.). Mammal Species of the World. A Taxonomic and Geographic Reference. USA: Johns Hopkins University Press; 2005.

18. Soriano PJ. Functional structure of bat communities in tropical rainforest and Andean Cloud Forest. Ecotropicos 2000; 13(1):1-20.

19. Ballesteros J, Racero J, Núñez M. Diversidad de murciélagos en cuatro localidades de la zona costanera del Departamento de Córdoba-Colombia. Rev MVZ Córdoba 2007; 12(2): 1013-1019.

20. Muñoz-Saba Y. Mamíferos. En: Rangel-Ch O (Ed.). Caracterización de flora y fauna de los humedales del Departamento de Córdoba. Bogotá: Universidad Nacional de Colombia (ICN); 2004.

21. Alberico M, Savedra CA, García-Paredes H. Murciélagos caseros de Cali (Valle del Cauca, Colombia). Caldasia 2005; 27(1):117-126.

22. Sampedro-Marín AC, Martínez CM. Recomendaciones para disminuir la convivencia del murciélago casero (Molossus molossus) con la población humana en la ciudad de Sincelejo, Departamento de Sucre, Colombia. Rev Colomb Cienc Anim 2009; 1(1):65-70.

23. Sampedro-Marín AC, Martínez-Bravo CM, Otero-Fuentes YL, Santos-Espinosa LM, Osorio-Ozuna S, Mercado-Ricardo AM. Presencia del murciélago casero (Molossus molossus pallas, 1776) en la ciudad de Sincelejo, departamento de Sucre, Colombia. Caldasia 2008; 30(2):495-503. 
24. Núñez C, Barona G, Astudillo M. Primeros hallazgos de Leptospiras en murciélagos en áreas urbanas del departamento del Valle. XXXVIII Congreso Nacional de Ciencias Biológicas, Armenia. Rev Asoc Col Cienc Biol, 2003.

25. Sampedro-Marín AC, Martínez C, De La Ossa K, Otero Y, Santos L, Osorio S, et al. Nuevos registros de especies de Murciélagos para el departamento de Sucre y algunos datos sobre su ecología en esta región colombiana. Caldasia 2007; 29(2):355-362.

26. Willig $M R$, Presley $S$ J, Bloch $C P$, Hice $C L$, Yanoviak SP, Díaz MM, et al. Phyllostomid bats of lowland Amazonian forest: effects of anthropogenic alteration of habitat. Biotropica 2007; 39:737-746.

27. Castro-Luna AA, Sosa VJ, Castillo-Campos G. Quantifying phyllostomid bats at different taxonomic levels as ecological indicators in a disturbed tropical forest. Acta Chiropt 2007; 9:219-228.
28. Medellín R, Equihua M, Amín M. Bat diversity and abundance as indicators of disturbance in Neotropical rainforests. Conserv Biol $2000 ; 14: 1666-1675$.

29. Ramírez-Chaves HE, Mejia-Egas O, Sambrano-G G. Anotaciones sobre dieta, estado reproductivo, actividad y tamaño de colonia del murciélago mastín común (Molossus molossus: Molossidae) en la zona urbana de Popayán, Departamento del Cauca, Colombia. Chiroptera Neotropical 2008; 14 (2):1-7.

30. Rydell J. Bats and their insect prey at streetlights. En: Long core, R.C (ed). Ecological consequences of artificial night Lighting. Washington: Island Press; 2006.

31. Ortega J, Castro-Arellano I. Artibeus jamaicensis. American Society of Mammalogists. Mammalian Species 2001; 662:1-9. 